\title{
Hydro-Mechanical Modelling of the Development of Preferential Gas Pathways in Claystone
}

Pierre Gerard, Jon Harrington, Robert Charlier, and Frédéric Collin

\begin{abstract}
During long-term repository of radioactive waste in argillaceous formation, the gas migration in the host formation and in the engineered barriers is a crucial issue, because it could affect the safety function of the clay barriers. Gas migration tests on Callovo-Oxfordian argillite have been performed by the British Geological Survey. The observations show that gas migration is often accompanied by the development of preferential pathways, which propagate through the sample. Hydro-mechanical numerical modelling of these tests is performed. It appears that a continuum approach to the movement of gas is not sufficient to reproduce the experimental observations. A coupling between deformation of the medium, permeability and air entry pressure is proposed. The numerical results show that such coupling play an important role in a successful simulation of such gas flows.
\end{abstract}

Keywords: gas migration, numerical modelling, preferential pathways, hydromechanical coupling, embedded fracture model.

\section{Introduction}

During long-term storage of radioactive waste disposal in clay-based rocks, steels containers will corrode. This two processes lead to the production of gas,

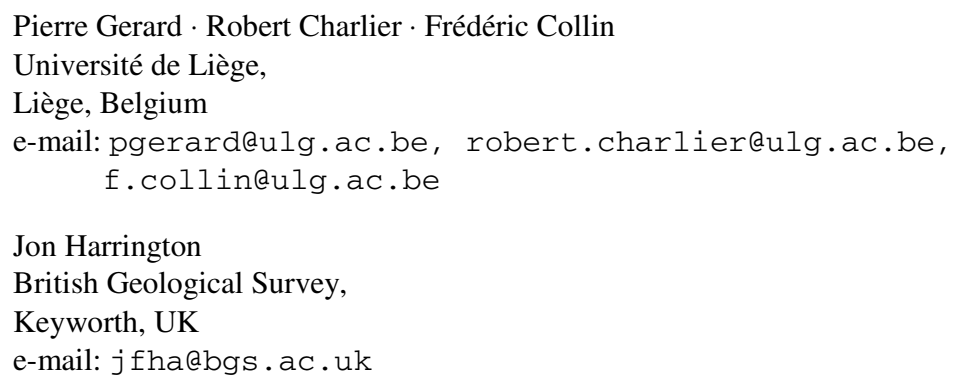


predominantly hydrogen. The understanding of the gas migration processes in the host formation and in the engineered barriers is a crucial issue, because it could affect the safety function of the clay barriers (Ortiz et al. 2002, Volckaert et al. 1995).

The transfer of gas has been studied experimentally, exhibiting complex behaviour in particular for gas flow under initially water saturated conditions. Long-term gas injection tests on argillaceous materials show flow instabilities, which are associated with the opening and the closing of localised pathways through the sample (Horseman et al. 1999, Harrington \& Horseman 2003, Marshall et al. 2005).

Such preferential pathways could not be reproduced with the classical models, based on a continuum biphasic approach of the flows. Initially soil heterogeneity can provide an explanation of such observations (Delahaye \& Alonso 2002). Another way to reproduce these flow instabilities numerically is to introduce an additional hydro-mechanical coupling between the permeability, the water retention curve and the strains (Olivella \& Alonso 2008). This second option is introduced in the finite element code Lagamine to reproduce observations obtained from a new long term gas injection test performed by the British Geological Survey on Callovo-Oxfordian argillite.

In this paper, the main experimental observations of the laboratory experiment are first described. The hydro-mechanical model is then presented, with a focus on the new hydro-mechanical coupling that takes into account the evolution of the permeability and the retention curve with the strains. The numerical results are detailed and the need of a strong hydro-mechanical coupling to reproduce the experimental observations is emphasized.

\section{Experiment Description}

A long term gas injection test has been performed by the British Geological Survey on Callovo-Oxfordian argillite. The permeameter developed by the British Geological Survey presents a novel feature: the use of porous annular guard-ring filters around the inflow and outflow filters, which allow the estimation of hydraulic anisotropy with a single test (Harrington et al, 2009). Prior to gas injection, each sample is restressed, resaturated and the baseline hydraulic properties (intrinsic permeability and specific storage) determined. During the gas injection phase, helium injection occurs through the base of the sample by slowly increasing gas pressure in a series of steps (from 6.5 to $10.5 \mathrm{MPa}$ ). A backpressure of $4.5 \mathrm{MPa}$ is continuously imposed at the top of the sample through the central filter and an isotropic confining stress of $12.5 \mathrm{MPa}$ is maintained during the entire experiment. The argillite specimen has an initial diameter of $54.4 \mathrm{~mm}$ and a height of 53.9 $\mathrm{mm}$. The orientation of the sample is perpendicular to the bedding plan. More details about the mineralogical composition of the Callovo-Oxfordian argillite can be found in Wileveau \& Bernier (2008). 
A complete analysis of the experimental results can be consulted in Harrington $\&$ Noy (2010). In this paper we present just the main observations, as the evolution of the gas outflow according to the injection gas pressure (Fig.1). With a fixed gas pressure of 6.5 and then 7.0 $\mathrm{MPa}$ applied to the injection filter, a small emergent flux is measured in the backpressure system. Gas pressure is then increase to 7.5 $\mathrm{MPa}$ and held constant. After a further 47 days (i.e. at an elapsed time of around 171 days), outflow begins to increase. At around 200 days there is a spontaneous increase in outflow, all of which are symptomatic of the development of conductive gas pathways. Some variation in gas discharge rates are then detected, which can be interpreted as evidence of dynamic pathway behavior. In their entirety, the observations reported by Harrington \& Noy (2010) are difficult to explain with standard porous medium flow models and suggest that a strong hydromechanical coupling is therefore needed to adequately explain the formation and subsequent behavior of gas conductive pathways within the Callovo-Oxfordian argillite.

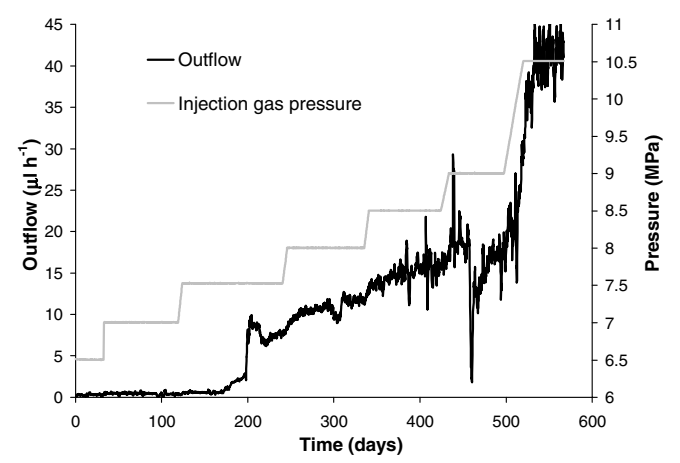

Fig. 1. Experimental gas outflow and gas injection pressure evolution plotted against time.

\section{Hydro-Mechanical Model}

To reproduce water and helium transfers in partially saturated porous media, a biphasic flow model is used. This model is comprised of a liquid phase, composed of liquid water and dissolved helium and a gaseous phase, which is an ideal mixture of dry helium and water vapour. It takes into account the advection of each phase using the Darcy's law and the diffusion of the components within each phase (Fick's law). More details about the biphasic flow model are available in Gerard et al. (2008). The retention curve and the water relative permeability curve are given by the van Genuchten's model (van Genuchten 1980). The gas relative permeability curve is a cubic function. The hydraulic parameters for argillite are presented in Table 1. The initial anisotropy of argillite is deduced from hydraulic tests performed before the injection of gas (Harrington \& Noy 2010). 
Table 1. Hydraulic and mechanical parameters for argillite

\begin{tabular}{lll|lll}
\hline $\mathrm{k}_{\text {hor }}$ & Horizontal intr. permeability $\left(\mathrm{m}^{2}\right)$ & $4 \times 10^{-20}$ & $\mathrm{E}_{0}$ & Young modulus (MPa) & 3800 \\
$\mathrm{k}_{\text {vert }}$ & Vertical intr. permeability $\left(\mathrm{m}^{2}\right)$ & $1.33 \times 10^{-20}$ & $v_{0}$ & Poisson's ratio (-) & 0.3 \\
$\Phi$ & Porosity (-) & 0.18 & $\mathrm{c}$ & Cohesion (MPa) & 3 \\
$\mathrm{~m}$ & Van Genuchten coefficient (-) & 0.55 & $\varphi$ & Friction angle ( $\left.{ }^{\circ}\right)$ & 20 \\
$\mathrm{n}$ & Van Genuchten coefficient (-) & 1.49 & $\mathrm{~b}$ & Biot's coefficient (-) & 0.6 \\
$\mathrm{P}_{\mathrm{r}}$ & Van Genuchten parameter $(\mathrm{MPa})$ & 15 & & & \\
\hline
\end{tabular}

In order to reproduce the development of preferential gas pathways in argillite, an evolution of the permeability and the air entry pressure with the strain is taken into account through the embedded fracture model proposed by Olivella \& Alonso (2008). The basic idea of this model is based on the appropriate representation of single fractures embedded in a continuous finite element matrix. These fractures are generated on tensile stress paths and are characterized by their aperture $b$. Permeability along the fracture $K_{i j}$ increases with the opening of these features, whilst the air entry pressure $P_{r}$ decreases (Olivella \& Alonso 2008, Gerard 2011):

$$
\begin{gathered}
K_{i j}=K_{\text {matrix }}+K_{0}\left(1+\lambda\left(\left\langle\varepsilon_{n}-\varepsilon_{0}\right\rangle\right)\right)^{3}\left(\delta_{i j}-n_{j} n_{i}\right) \\
P_{r}=P_{r, 0} \frac{1}{1+\lambda\left(\left\langle\varepsilon_{n}-\varepsilon_{0}\right\rangle\right)}
\end{gathered}
$$

with $\varepsilon_{n}$ the normal strain to the fracture, $\varepsilon_{0}$ the threshold strain required to initiate fracture opening, $\lambda$ a parameter reflecting the density of fractures and the rugosity, $K_{\text {matrix }}$ the permeability of the undisturbed argillite, $K_{0}$ the initial permeability along the fracture and $\underline{n}$ the normal to the fracture (considered parallel to the maximum principal strain $\varepsilon_{l}$ ). The mechanical model of argillite is a nonassociated linear elastic-perfectly plastic model (with Van Eekelen yield surface). This model is written in terms of net stress and the mechanical parameters of argillite are presented in Table 1.

\section{Boundary Value Problem and Numerical Results}

A 2D axisymetrical model of the gas injection test is performed with the finite element code Lagamine. The geometry and the initial conditions of the problem are presented on Fig.2. A water pressure of $4.5 \mathrm{MPa}$ is maintained at the backpressure filter. The gas pressure is increased in a stepwise manner at the injection filter. Due to the isotropic confining stress $(12.5 \mathrm{MPa})$, the strain state remains practically homogeneous in the sample during all of the gas injection stages. An initial fracture must therefore be defined in the sample (Fig.3) which corresponds to the zone where the development of conductive pathways is possible. This fracture is initially closed due to the confining stress, but will be then be allowed to open as gas pressure in the sample increases. 


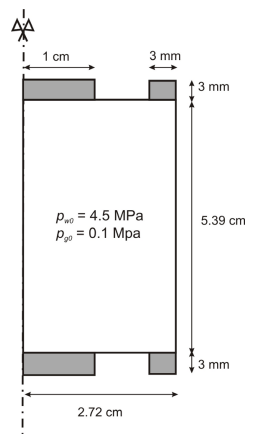

Fig. 2. Geometry of the problem.

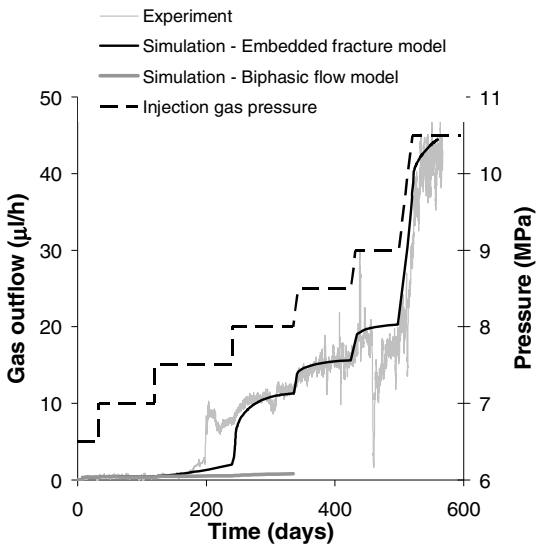

Fig. 4. Gas outflow: comparison between experimental and numerical results with or without embedded fracture model.

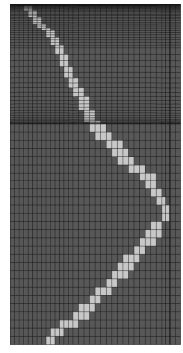

Fig. 3. Initial fracture in sample.

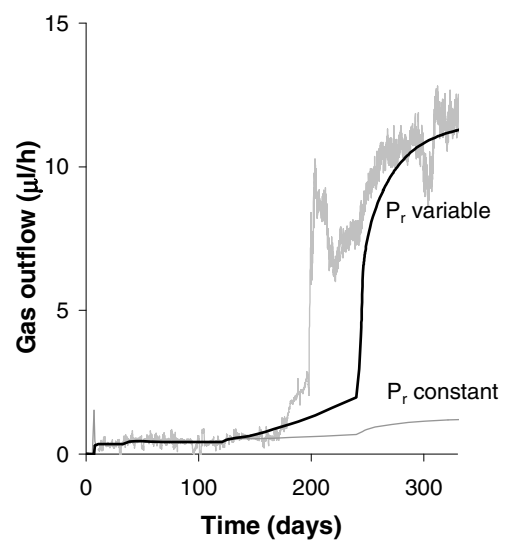

Fig. 5. Gas outflow: comparison between experimental and numerical results with embedded fracture model and with or without air entry pressure evolution.

The numerical results show that a continuous biphasic flow model allows the reproduction of the gas outflow during the first gas increments, but is not able to explain many aspects of the transfer processes during breakthrough (Fig.4). In contrast, using the embedded fracture model $\left(\lambda=5000 ; \varepsilon_{0}=-6.410^{-4}\right)$ provides a good reproduction of the experimental results when the development of preferential pathways begins (Fig.4). The onset of the breakthrough is nevertheless not particularly well modelled by this approach, because the development of pathways is obtained numerically at each gas pressure increment. Fig.5 shows how a decrease in the air entry pressure coupled with the strain is needed to obtain the sudden and spontaneous increase in gas outflow. 


\section{Conclusions}

A 2D axisymetrical model of a gas injection test on Callovo-Oxfordian argillite has been performed. A strong hydro-mechanical coupling is introduced through the introduction of an embedded fracture model. Evolution of permeability and air entry pressure is induced by changes in fracture aperture and its coupling with strain. The numerical results show the need for such hydro-mechanical models, in order to better reproduce the development of conductive gas pathways. The preferential desaturation along the fractures induced by the decrease of the air entry pressure is particularly important in order to obtain the sudden and large gas outflow observed during major gas breakthrough. It must be mentioned that gas flow is likely to be along more than one pathway. The simplification of the model to one feature may also explain some of the observed discrepancies.

A sensitivity analysis on the geometry of the initial fracture in the sample, but also on the calibration of the embedded fracture model, should be presented in a next paper. This model now needs to be tested on the response of the argillite to stepwise decrements in gas pressure, including the 'shut-in' phase of the test, when gas injection ceases and the pressure is allowed to decay to a threshold value.

\section{References}

Delahaye, C.H., Alonso, E.E.: Soil heterogeneity and preferential paths for gas migration. Eng. Geol. 64, 251-271 (2002)

Gerard, P., Charlier, R., Barnichon, J.D., Su, K., Shao, J.F., Duveau, G., Giot, R., Chavant, C., Collin, F.: Numerical modelling of coupled mechanics and gas transfer around radioactive waste in long-term storage. J. Theor. Appl. Mech. 38(1-2), 25-44 (2008)

Gerard, P.: Impact des transferts de gaz sur le comportement poro-mécanique des matériaux argileux. Thèse de doctorat. Université de Liège, Belgium (2011)

Harrington, J.F., Horseman, S.T.: Gas migration in KBS-3 buffer bentonite. Sensitivity of test parameters to experimental boundary conditions. Technical Report SKB-TR-03-02, p. 57 (2003)

Harrington, J.F., Noy, D.J.: Gas and water flow in the Callovo-Oxfordian argillite: Progress, Technical Report British Geological Survey CR/10/101 (2010)

Harrington, J.F., Noy, D.J., Horseman, S.T., Birchall, J., Chadwick, R.: Laboratory study of gas and water flow in the Nordland Shale, Sleipner, North Sea. In: Grobe, M., Pashin, J.C., Dodge, R.L. (eds.) Carbon Dioxide Sequestration in Geological Media-State of the Science, pp. 521-543 (2009)

Horseman, S.T., Harrington, J.F., Sellin, P.: Gas migration in clay barriers. Eng. Geol. 54, 139-149 (1999)

Marschall, P., Horseman, S.T., Gimmi, T.: Characterisation of Gas Transport Properties of the Opalinus Clay, a Potential Host Rock Formation for Radioactive Waste Disposal. Oil Gas Sci. Technol. 60, 121-139 (2005)

Olivella, S., Alonso, E.E.: Gas flow through clay barriers. Géotechnique 58(3), 157-168 (2008)

Ortiz, L., Volckaert, G., Mallants, D.: Gas generation and migration in clay barriers. Eng. Geol. 64, 287-296 (2002)

Van Genuchten, M.T.: A closed-form equation for predicting the hydraulic conductivity of unsaturated soils. Soil Sci. Soc. Am. J. 44, 892-898 (1980)

Volckaert, G., Ortiz, L., de Cannière, P., Put, M., Horseman, S.T., Harrington, J.F., Fioravante, V., Impey, M.: MEGAS: Modelling and Experiments on Gas Migration in Repository Host Rocks - Phase 1 Final Report. European Commission Report EUR 16235 EN (1995) 